other self-categorization theorists (e.g., Turner et al. 1994), this belief has informed most of our research for the last twenty years. Accordingly, it was used as a basis for critiquing the large body of social cognitive research into stereotyping and for developing and testing an alternative metatheoretical perspective. This argues that stereotyping and related group processes are not the product of cognitive bias or collective irrationality, but of adaptive sensemaking processes which serve: (a) to represent important social realities from the perspective of membership in particular social groups, and (b) to allow those groups to engage in meaningful forms of social action.

An example is provided by research into inter-category accentuation, that is, the tendency to represent distinct groups in blackand-white terms rather than shades of grey (e.g., Krueger \& Rothbart 1990; Tajfel 1969). Haslam and Tumer (1992; 1998) suggest that, rather than representing a distortion of the individual properties of stimuli, such accentuation serves to represent veridically their higher-order properties as members of social groups perceived from a particular vantage point. We argued that such judgments only appear distorted if one accepts the individualistic doctrine that the truth about people resides in their individuality, and if one privileges a single perspective (e.g., that of the researcher or "independent" judge) in one's appraisal of accuracy. In contrast, self-categorization theorists argue that there are higher-order social realities and distinct social and political perspectives, which stereotypes and social judgments need to represent, if they are to allow the perceiver to function effectively in the social world (see Oakes et al. 1994; Spears et al. 1997). It is not hard, for example, to see why it would have been problematic - not just psychologically, but also politically - for Black South Africans in the Apartheid era to see all South Africans as individuals and to accept the "objective" judgments of the white judiciary, which sought to invalidate perceptions that were consonant with Black Consciousness. Haslam and Tumer (1998) used the following court exchange involving the Black activist Steve Biko to illustrate this point:

Judge Boshoff: But now why do you refer to you people as blacks? Why not brown people? I mean you people are more brown than black.

Biko: In the same way as I think white people are more pink and yellow and pale than white.

[General laughterer in the court]

Judge Boshoff: Quite ... but now why do you not use the word brown then?

Biko: No, I think really, historically, we have been defined as black people, and when we reject the term non-white and take upon ourselves the right to call ourselves what we think we are, we have got available in front of us a whole number of alternatives,,$\ldots$. and we choose this one precisely because: we feel it is most accomnodating. (Biko 1978/1988, p. 121)

In treating Biko as a fool, the judge here takes the line of most cognitive social psychologists in suggesting that accentuated judgment (seeing things as black-and-white rather than brown-andpink) misrepresents reality by exaggerating its true nature. But, providing we share Biko's political goals, we can see that it is the judge who is the fool.

Yet, while there are important points of contact between the work of self-categorization theorists and the arguments of $K \& F$, we would note that there are still significant residual differences. Most pointedly, we do not believe that the bias agenda has arisen primarily as a result of social psychologists' failure to survey a full range of behavioural responses, and hence, that it will be remedied by statistical or other strategies that attempt to correct for this limited sampling. Like social cognitivists, Judge Boshoff was not at fault because he did not have access to enough information of the right sort. Indeed, if he had had more information, it seems likely that (from our perspective) he would have interpreted that incorrectly, as well. Instead, then, the primary problem lies in his very limited interpretation of the data that he already had access to. And what is driving this? Problems of negative emphasis? Of non-Bayesian inference? Of lack of balance?
It is none of these. Rather, we can see that the limitations of the judge's perspective were a direct reflection of his in-group's ideology and political imperatives. Likewise, in social psychology, the bias agenda can be traced to ideological priorities which reify a particular definition of social reality - one which sees the truth about people (whether perceivers or perceived) as lying in their status as isolated individuals, rather than as members of functioning social groups who need to act in relation to a specific social context (Oakes et al. 2001; Turner \& Oakes 1997).

Significantly too, it is apparent that in K\&F's own Utopian future they still retain the belief that there is a single transcontextual reality, which can be uncovered by appropriate statistical and behavioral testing. Psychologically, this conviction seems highly questionable. On political grounds, we are generally motivated to favour one version of social reality over another (i.e., ours) and to present this as the truth, but in order to do justice to social psychology, we need to understand that the social world is comprised of multiple realities. So, although as political agents we may favour Biko's version of reality over Boshoff's, in order to make progress as social psychologists we need to understand that, for the people and groups who endorse such worldviews, there are competing realities here. In short, the path to progress lies in an appreciation of the interplay between psychology and social context that creates these realities, rather than in attempting to achieve some artificial balance in a decontextualized psychology.

The same, incidentally, is true of classic studies of visual perception. To make sense of what happens in an Ames' room, for example, we need to understand that the visual world really is different for participants and for detached observers. In research of this form, of course, there is no debate about which of these two worlds to privilege when labeling one set of perceptions "right" and the other "wrong," and so we have no political difficulty achieving a "balanced" psychology of perception. But the social world typically isn't like this - as members of different social groups we have different values, norms, ideologies, and cultures. In other words, we have different social perspectives. Moreover, as the history of social cognition research demonstrates, when the differences between these are downplayed, it is the values and perspective of more powerful groups that tend to be privileged in arbitrating over error and accuracy, and the balance between the two (Hopkins et al. 1997; Spears \& Smith 2000).

So, as $\mathrm{K} \& \mathrm{~F}$ suggest, let us celebrate social psychology as veridi$\mathrm{cal}$ and adaptive, rather than error-prone and error-ridden. But let us accept that this requires an appreciation of differences in social perspective and in associated psychological truths - as well as appreciation of the political and sooiostructural reasons for these differences - rather than an a priori commitment to balance. If we do not, we suspect that social psychology will continue to lose its way in an array: of baffling conundra and seemingly paradoxical phenomena, and will simply substitute one set of problems for another. For when the labels "truth" and "error" are attached to different phenomena by members of different groups, methodological criteria alone will never resolve the thorny questions of how much balance is enough, and who has the right to decide.

\section{Out of the theoretical cul-de-sac}

\section{Ralph Hertwig and Annika Wallin}

Center for Adaptive Behavior and Cognition, Max. Planck Institute for Human Development, 14195 Berlin, Germany. hertwig@mpib-berlin.mpg.de wallin@mpib-berlin.mpg.de

Abstract: A key premise of the heuristics-and-biases program is that heuristics are "quite useful." Let us now pay more than lip service to this premise, and analyse the environmental structures that make heuristics more or less useful. Let us also strike from the long list of biases those phenomena that are not biases and explore to what degree those that remain are adaptive or can be understood as by-products of adaptive mechanisms. 
Let us waste no more time quarrelling over the diagnosis. Krueger $\&$ Funder $(\mathrm{K} \& \mathrm{~F})$ are right. Social psychology and related fields have oversold violations of behavioural and cognitive norms. This state of affairs was foreseeable, and not only with the benefit of hindsight. For instance, back in 1982, Kahneman and Tversky explicitly acknowledged that "although errors of judgment are but a method by which some cognitive processes are studied, the method has become a significant part of the message" (1982, p. 124). Since then, the method has become the most significant part of the message.

It is thus high time the message that human reasoning is "ludicrous," "indefensible," and "self-defeating" be counterbalanced. But balance is not the only reason to rethink social psychology's research agenda. Even more important, as $K \& F$ point out, is the fact that the hunt for behavioural and cognitive flaws has led us to a cul-de-sac. Discovering another bias, error, violation, or illusion is a much less original, let alone theoretically fruitful, contribution today than it was 20 or 30 years ago. $K \& F$ list a number of promising routes out of the dead end - we add some related ones.

Let us at last pay more than lip service to a key premise of the heuristics and biases program that Tversky and Kahneman (1973) articulated in their original framing of the availability heuristic:

Availability is an ecologically valid clue for the judgment of frequency because, in general, frequent events are easier to recall or imagine than infrequent ones. (p. 209, our emphasis)

Assuming availability is ecologically rational (rather than irrational), how the heuristic reflects the structure of the environment should have been explored, but it was not. Instead, since the heuristic was proposed 30 years ago, countless papers have implicated it in countless biases - illusory correlations, unwarranted optimism, eyewitness identification errors, discriminatory biases, and hindsight bias, to name just a few. To the best of our knowledge, however, not a single paper has fleshed out how the availability heuristic may exploit ecological texture to estimate event frequencies, although this kind of analysis is precisely what is needed to predict the conditions under which it succeeds - and fails. The research program on fast and frugal heuristics demonstrates how the mapping between heuristics and environmental texture can be analysed (Gigerenzer et al. 1999). There is no reason why the heuristics to which many biases have been attributed cannot be subjected to such analysis, even if it requires more clarity about the underlying processes.

There is another, related route to change. This is to examine the long list of cognitive biases by asking the following three questions about each one.

Is the bias really a bias? There are several reasons why a cognitive phenomenon might have to be taken off the list of biases. Take the conjunction fallacy as an example. Virtually no one doubts that Tversky and Kahneman's (1983) Stanford undergraduates violated the conjunction rule when they judged Linda to be more likely a feminist and bank teller than only a bank teller. But does that mean that their students committed the conjunction fallacy? No. Semantic and pragmatic ambiguity led many of them not to reason according to the conjunction rule. In particular, the students had to infer what the experimenters meant by semantically and pragmatically ambiguous words such as probability and and. In doing so, they may have arrived at legitimate meanings that differ from mathematical probability (Hertwig \& Gigerenzer 1999) and logical AND (for different views on this issue, see Mellers et al. 2001). It is ironic that while many psychologists continue to interpret the outcome of semantic and pragmatic inferences as evidence of biased reasoning, others struggle to design artificial agents capable of inferring, for instance, which of multiple meanings of a polysemous word is appropriate in a given context. To them, designing systems that can "process language as skillfully as we do will signal the arrival of truly intelligent machines" (Jurafsky \& Martin 2000, p. 6).

Is the "blas" a design flaw or a built-in adaptation? Several researchers have recently argued that biases in (social) judgments may be design features rather than design flaws of the human mind (e.g., Haselton \& Buss 2003; Nettle 2004). Take, for example, Bjorklund's (1997) argument regarding childrèn's overconfidence in their competence. Children appear to misjudge their abilities on a broad range of cognitive tasks. How might such systematic miscalibration be adaptive? Bjorklund proposed that overrating one's ability has motivational benefits at a point in development at which one's behavioural and cognitive repertoires are extremely limited, and each novel task could be daunting. If children in this situation "rationally" assessed the difficulty of a task and their task-related skills, trying their hand only if they appeared to have the requisite skills, then they would never explore many novel tasks and territories. In fact, by avoiding tasks likely to overtax their skills, children would miss out on important opportunities to learn new things.

Is the "bias" a cheap price to pay for an adaptive mechanism? Even if a bias is not an adaptive feature, it may be a by-product of an adaptive mechanism. Take the hindsight bias as an example: Many researchers have stressed its detrimental consequences (e.g., Fischhoff 1982). In a recent model of the processes underlying the hindsight bias, Hoffrage et al. (2000) suggested that the hindsight bias is a by-product of a memory system that updates information constantly and automatically. Specifically, the model assumes that new information regarding the outcome of an event leads to an updating of the knowledge (cues) on which people's original evaluation of the event was based. When people attempt to reconstruct their original judgment, they access the updated knowledge base, opening the door to hindsight bias.

Knowledge updating is adaptive in that it prevents us from using information that, because of changes in the environment, may be outdated. It has a by-product - the hindsight bias. The bias, however, may be a relatively low price to pay for keeping the knowledge in our limited memory up-to-date. Consistent with this view, Hertwig et al. (2003) found that although updating can result in erroneous memories of past judgments (i.e., the hindsight bias), it increases the accuracy of future inferences.

Admittedly, claims about the adaptive nature of either biases or the processes that result in biases need to be carefully scrutinized. But they serve to emphasize that the design features of the human mind, like those of the human body, reflect trade-offs between benefits and costs. It is high time that we accept this simple truth about human cognition, and at last try to understand these tradeoffs, rather than dubbing them biases and calling it a day.

\section{Asch and the balance of values}

\author{
Bert H. Hodges \\ Department of Psychology, Gordon College, Wenham, MA 01984. \\ hodges@gordon.edu
}

Abstract: Values will be central to developing a more balanced social psychology. A nonconformist account of Asch's (1956) experiments is used to illustrate the role of multiple values and to support and extend Krueger \& Funder's (K\&F's) claims. A balance of values, one that goes beyond accuracy and truth, and that avoids absolutism and relativism, is needed.

Krueger \& Funder's (K\&F's) call for a more balanced social psychology is a challenge to be welcomed. My comments, intended to support and sharpen their claims, will focus on values, which they suggest will require renewed attention if balance is to be achieved (sect. 5). First, a "nonconformist" account of Asch's (1956) studies will be offered to illustrate K\&F's criticisms and recommendations. Second, some difficulties for addressing values will be briefly noted.

Contra K\&F (sect. 2.2.2), Asch designed his experiment precisely to counter the view that people are "sheep" (Cialdini \& Trost 1998). He thought that if there was unambiguous physical information available, people should and would say what they saw with- 